\title{
Probing the Jet Physics of TeV Blazars
}

\section{Ulisses Barres de Almeida*; Paula M. Chadwick, Sam J. Nolan, Martin Ward, T. J. Lowry McComb}

University of Durham, $U K$

E-mail: u.b.almeida@durham.ac.uk

We present serendipitous data from two independent observing campaigns on PKS 2155-304 in August and September 2003. The dataset comprises published TeV H.E.S.S. observations and optical polarimetric measurements from the Pico dos Dias Observatory in Brazil, spanning a few nights of simultaneous observations in both months. The source was close to its quiescent level during these observations and despite the limited resolution and extent of the contemporaneous data, the gamma-ray variability is clearly accompanied by activity of the optical polarized flux. Gamma-ray observations simultaneous with optical polarimetric measurements are rare in the literature, and a larger data-set from another campaign following the 2006 large VHE flare is to be published in a future work. Here we will describe the relationship between the optical and VHE observations and discuss the main motivations for using low-energy polarisation measurements in multi-wavelength campaigns involving high-energy observations.

Workshop on Blazar Variability across the Electromagnetic Spectrum

April 22-25 2008

Palaiseau, France

\footnotetext{
* Speaker.

${ }^{\dagger}$ U. Barres de Almeida acknowledges a Ph.D. grant from the CAPES Foundation, Ministry of Education of Brazil, for supporting this work. We also thank Drs. Z. Abraham and T. Dominici for kindly giving us access to the optical data used in this work prior to its first publication in these proceedings.
} 


\section{Introduction}

Blazars are the only group of extragalactic sources known to emit as a class in the VHE gamma-ray range $(\mathrm{E}>0.1 \mathrm{GeV})$. From these, all but one of the detected sources are HBLs (highfrequency peaked BL Lacs), characterised by a double-peaked non-thermal spectral energy distribution (SED) spanning the entire EM spectrum, and peaking respectively at soft X-rays and $\mathrm{GeV} / \mathrm{TeV}$ gamma-rays. The defining property of blazars, believed fundamental for rendering them capable of VHE emission, is the close alignment of their jets to the observer's line of sight. This geometrical configuration favours the Doppler boosting of the radiation emitted by the relativistic particles traveling down the jet and alleviates the severe restrictions on the escape of the VHE gamma-rays from the dense radiation fields of the inner jet and AGN regions imposed by photonphoton pair-creation (Maraschi et al. 1992). The nearby $(\mathrm{z}=0.116)$ southern source PKS 2155-304 is one of the brightest and most extreme BL Lacs in the sky; it was one of the first extragalactic VHE sources detected (Chadwick et al. 1999) and is now the primary AGN target for H.E.S.S. (High Energy Stereoscopic System) with which it has been observed since 2002 while in a wide range of states; a historical account of these observations can be found in a review by Punch (2007).

PKS 2155-304 has been targeted by several multi-wavelength (MWL) campaigns down the years. These campaigns have revealed broadband variability on timescales from years to minutes, the most dramatic episodes being registered recently in very-high energies. The main focus of these campaigns was establishing variability correlations along the many SED bands, since these are the defining tests for competing jet and emission models. While the correlation between X-rays and UV and X-rays and gamma-rays has been tested in great detail (e.g. Edelson et al. 1995, Urry et al. 1997 and Aharonian et al. 2005), the relation between the optical and the higher energy emission is still uncertain due to the difficulty in isolating the non-thermal optical emission from the jet from the rest of the thermal contribution. Polarisation measurements are ideal for this purpose since the non-thermal emission is expected to originate as synchrotron radiation from the particle populations in the jet. In fact, long-term studies of a large sample of BL Lacs have revealed the polarization fraction of the emission to be highly variable on all timescales (Ballard et al. 1990) and not necessarily follow the behaviour of the total flux (Smith 1996). Recent MWL studies on other sources have already revealed the polarised emission to bare some relation with X-ray radiation (Marscher et al. 2008), but more extensive probes of this correlation and its association to the highenergy gamma-ray bump lack and are necessary to support the leptonic scenarios (Maraschi et al. 1992, Sikora et al. 1994) favoured by the aforementioned UV/X-ray and X-/gamma-ray studies.

\section{Observations and Results}

The data presented here comprises two sets of quasi-simultaneous serendipitous optical polarimetric and VHE observations, taken independently during a few nights in the months of August and September 2003. The VHE gamma-ray observations were carried out by H.E.S.S. whilst the array was still under construction, and therefore the stereoscopic data shown are for 2 or 3-telescope configurations; this means that the lower energy-threshold of the observations is higher than for current H.E.S.S. data $(\sim 300 \mathrm{GeV})$ and the temporal resolution attainable is limited to daily averages by the more restricted photon-count statistics of the experiment at the time. The source was 

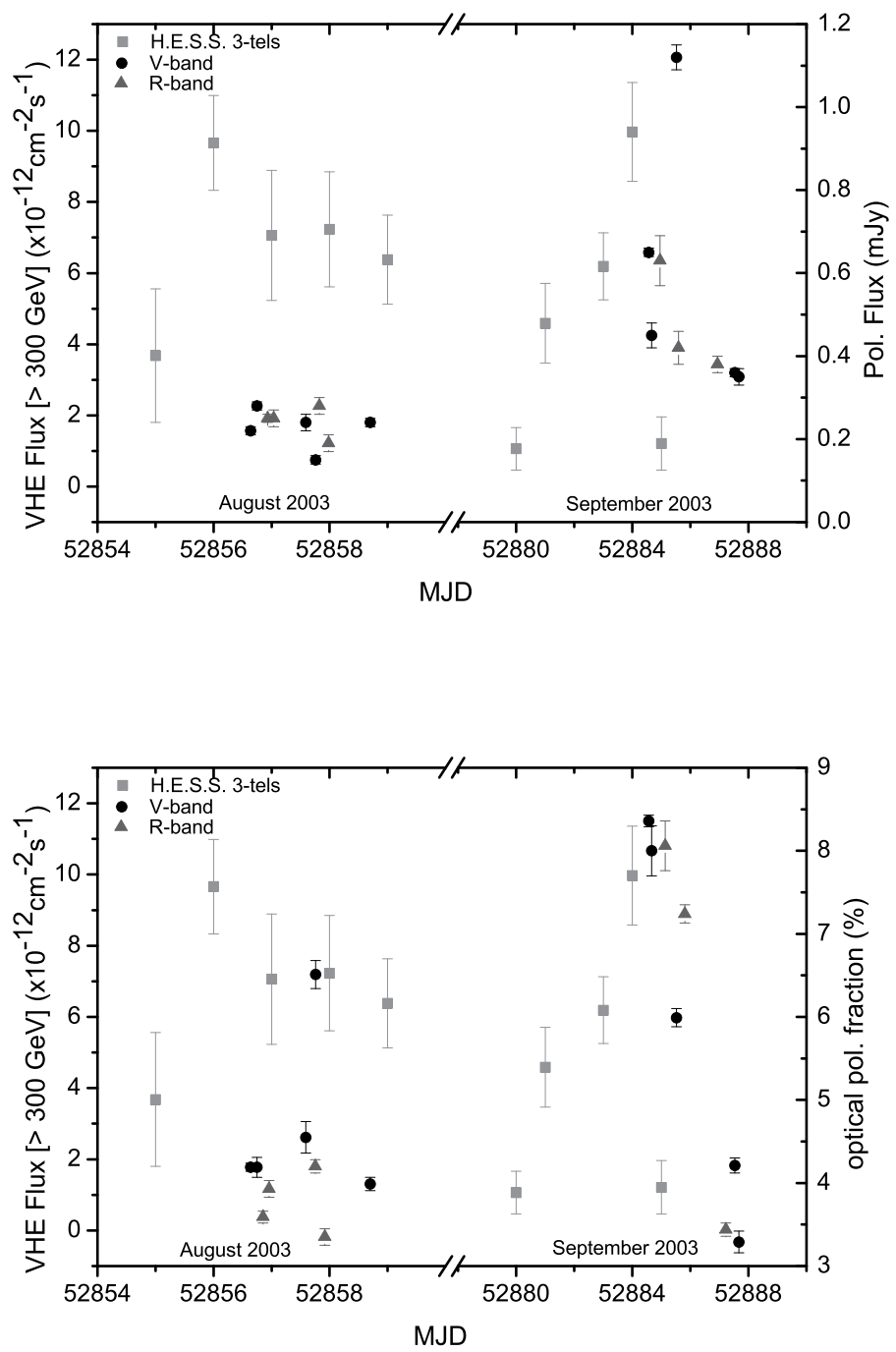

Figure 1: The TeV light curve of PKS 2155-304 during August-September 2003, derived from H.E.S.S. observations. The top panel shows contemporaneous measurements of the optical polarised emission, and the bottom panel shows the corresponding variations in the degree of optical polarisation. Optical data are derived from the Pico dos Dias OPD/LNA observatory.

in a relatively low state during the observations, the average flux for the period contemporaneous to the optical data being of the order of $7 \times 10^{-12} \mathrm{~cm}^{-2} \mathrm{~s}^{-1}$, but clear inter-day variability was seen, with amplitudes of up to $8 \times 10^{-12} \mathrm{~cm}^{-2} \mathrm{~s}^{-1}$; the complete dataset and analysis are presented in an earlier paper by Aharonian et al. (2005). The multi-band optical polarization measurements were carried out with the imaging polarimeter IAGPOL (Magalhães et al. 1996), coupled to the $0.6 \mathrm{~m}$ Boller \& Chivens telescope at Pico dos Dias observatory (LNA/OPD), in Brazil. The data presented here include only a small fraction of a 3-year campaign, the complete results of which 
are published for the first time in these proceedings by Dominici et al. ${ }^{1}$

The light-curve for both datasets is presented in Fig. 1, which shows the contemporaneous $\mathrm{TeV}$ and polarimetric data for MJDs 52854-52858 and 52880-52888. The TeV data for August are mostly consistent with constant flux; the September data nevertheless show variability, and a $\mathrm{TeV}$ flare is clearly followed in its entire development through the 5 nights of observations. The restricted dataset and the lack of simultaneity prevent the drawing of extensive conclusions, but some observations of more general character can be made.

In parallel with the VHE data, polarisation measurements in both $\mathrm{V}$ and $\mathrm{R}$ bands are presented in the light-curves of Fig 1 (top panel); the polarized flux in both bands shows no intense variability during August, but it appears to reflect the TeV activity in September, when an optical flare is seen in the V-band polarimetric data, following the gamma-rays with a 2-day lag (in the R-band only the decaying trend of the flare is seen). Since there are no further VHE observations immediately after these data, the association between the optical and gamma-ray features is only tentative. The total optical flux is not presented here but behaves similarly, doubling its value in the V-band two nights after the VHE emission reaches its maximum.

More interesting to note is the behaviour of the percentage of polarisation of the optical flux in Fig. 1 (bottom panel), which does not follow the optical flux light-curve (a rather common behaviour as noticed by Smith 1996), but better traces the VHE emission. In fact, the September data appear to closely follow the TeV flux, as the downward trend of the optical emission is seen following quasi-simultaneously the decay of the gamma-ray flare. In August the V-band polarisation fraction varies by a factor of 3 in one hour, with little counterpart in the R-band and no counterparts in the gamma-ray flux.

Despite the relative paucity of the data, which prevents more robust analysis to be performed, observations appear to support correlations in the trend between the $\mathrm{TeV}$ and the optical polarisation light-curves; in particular, the VHE flare in September seems to carry a firm counterpart from the optical side, even though it isn't completely sampled nor can detailed temporal relations be established. These results are similar to the ones presented by Joshi et al. (2000) on the BL Lac object Mkn 501, and suggest a close connection between the optical synchrotron and the TeV photons, which is consistent with both emissions having a common spatial origin in the jet, as expected in the leptonic scenarios for the origin of the VHE gamma-rays within the framework of synchrotron-self Compton (SSC) models.

\section{Radio Polarisation Counterpart}

The contemporaneous optical data presented by Dominici et al. (2008) show that the electric vector position angle (EVPA) was nearly constant during the observations, varying within the narrow range of $\sim 60^{\circ}-65^{\circ}$ in both August and September; on MJD 52858 a $\sim 25^{\circ}$ rotation was seen in the period of one day, but lack of observations after this event prevented the detection of any counterparts to it.

In addition to the optical and VHE data, a single-epoch contemporaneous VLBA polarisation measurement is available for PKS 2155-304 on 20 September (MJD 52902), just 10 days after

\footnotetext{
${ }^{1}$ There exist further contemporaneous VHE/optical measurements from these campaigns, but they are reserved for more detailed future analysis and will be published elsewhere.
} 
the last data in Fig. 1. The measurement was made at $15.4 \mathrm{GHz}$ with the Very Large Baseline Array (VLBA), and shows the radio emission to have a degree of polarisation varying spatially along the core-component between $\sim 3-10 \%$, (similar to the values observed in optical) and an electric vector position angle (EVPA) of $131^{\circ}$, misaligned by $30^{\circ}$ to the innermost jet position angle (Piner et al. 2008). The radio and optical EVPAs are almost orthogonal to each other (in fact $\chi_{\text {core }}-\chi_{o p t} \sim 70^{\circ}$ ), and while the former is almost aligned to the jet direction, indicative of emission originating in an optically thin shocked region (presenting transverse magnetic lines), the optical EVPA is perpendicular to it. Given the expected alignment between both EVPA measurements (Jorstad et al. 2007) this relation would suggest either a different spatial origin from the $\mathrm{mm}$ VLBA core for the optical (and consequently VHE) emissions or, more probably, that it comes from an unresolved compact component or region within the radio core. The $7 \mathrm{~mm}$ radio map also registers one additional jet component for which no significant polarised flux is detected. At the time of these measurements the VHE flux was $\sim 1.4 \times 10^{-11} \mathrm{~cm}^{-2} \mathrm{~s}^{-1}$, similar to the previous months, with no appreciable variability being registered.

\section{Motivation for Future Observations}

Multi-wavelength observations constitute the preferred method for probing the jet physics of blazars and AGNs in general. By studying the correlations between multiple emission bands of their SED one can constrain the mechanisms responsible for the production of radiation; in particular, it is important to look simultaneously at both SED bumps to understand how the low- and high-energy emissions relate to each other. For BL Lacs this means the inclusion of GeV or TeV observations in the campaign. Such a program is relatively easy to carry out using UV/X-ray data and gamma-rays (specially with the current generation of ground-based Cherenkov Telescopes) and are the usual practice providing us with the relevant timescales for the source and the upper limits on the sizes of the emitting regions. They are nevertheless unable to locate the sites of emission within the source in a model-independent way. For this, it is necessary to establish a connection between the interferometric maps and the high energy emission; this can be done by studying the optical/radio correlations of the polarised emission (Gabuzda et al. 2006, Jorstad et al. 2007) which allows one to link the structures in the radio maps with specific features in the light-curve, that can then be traced all the way up to the high energies (Marscher \& Jorstad 2007).

Nevertheless, the use of optical or radio polarimetric data in multi-wavelength campaigns which include gamma-ray information is rare, and this work is the first since the one by Joshi et al. (2000) to present near-simultaneous VHE and optical polarisation light-curves; data from a larger optical polarisation/VHE campaign of PKS 2155-304 is also under analysis at the moment and will be published in a future work. By presenting these preliminary results we wish to advocate the regular use of polarisation measurements, both in radio and VLBI, in conjunction with VHE gamma-ray observations, as the preferred method of studying the sites of particle acceleration in extragalactic AGN jets and probing its emission mechanisms. The recent launch of the gamma-ray satellite GLAST (Gamma Large Area Space Telescope) also promises to facilitate the development of such studies. 


\section{References}

[1] F. Aharonian et al. (H.E.S.S. Collaboration) 2005, H.E.S.S. Observations of PKS 2155-304, A\&A 430 (865).

[2] F. Aharonian et al (H.E.S.S. Collaboration) 2005, Multi-wavelength observations of PKS 2155-304 with HESS, A\&A 442 (895).

[3] K.R. Ballard, A.R.G. Mead, P.W.J.L. Brand and J.H. Hough 1990, The optical and infrared emission of blazars, MNRAS 243 (640).

[4] P.M. Chadwick et al. 1999, Very High Energy Gamma Rays from PKS 2155-304, ApJ 513 (161).

[5] P. Charlot, D. Gabuzda, H. Sol, B. Degrange and E. Pare 2000, VLBI polarization observations of Mrk 421 coordinated with TeV gamma-ray monitoring by the CAT telescope, in proceedings of Texas Symposium on Relativistic Astrophysics and Cosmology NuPhS (Vol . 80).

[6] T.P. Dominici, Z. Abraham, A.M. Magalhaes et al. 2008, Optical polarization variability in TeV BL Lacs, this proceedings.

[7] R. Edelson, J. Krolik, G. Madejski et al. 1995, Multiwavelength monitoring of the BL Lacertae object PKS 2155-304. 4: Multiwavelength analysis, ApJ 438 (120).

[8] D.C. Gabuzda, M.L. Sitko,P.S., Smith 1996, Correlations Between the VLBI and Optical Polarization of BL Lacertae Objects, AJ 112 (1887).

[9] D.C. Gabuzda, and J.L. Gomez 2001, VSOP polarization observations of the BL Lacertae object OJ 287, MNRAS 320 (L49).

[10] D.C. Gabuzda 2003, VLBI Polarisation Properties of a Complete Sample of Radio-Loud BL Lacs, ASS 288 (39).

[11] S.V. Jorstad, A.P. Marscher et al. 2007, Multiwaveband Polarimetric Observations of 15 Active Galactic Nuclei at High Frequencies: Correlated Polarization Behaviour, AJ 134 (799).

[12] U.C. Joshi, K.S. Baliyan, S. Ganesh, M.R. Deshpande et al. 2000, Coordinated TeV gamma-ray and optical polarization study of BL Lac object Mkn 501,Bull. Astr. Soc. India 28 (409).

[13] A.M.. Magalhaes et al. 1996, High Precision CCD Imaging Polarimetry, in proceedings of Polarimetry of the interstellar medium ASP Conf Series(97).

[14] L. Maraschi et al., G. Ghisellini, A. Celotti 1992, A jet model for the gamma-ray emitting blazar 3C 279, ApJ 397 (L5).

[15] A.M. Marscher, S. Jorstad 2007, Use of Multiwaveband Polarization and Light Curves to Identify Sites of Gamma-ray Emission in Blazar Jets, in proceedings of The Fisrt GLAST Symposium AIP Conf Series(921).

[16] A.P. Marscher, S.G. Jorstad et al. 2008, The inner jet of an active galactic nucleus as revealed by a radio-to-gamma-ray outburst, Nature 452 (966).

[17] B.G. Pinner, N. Pant and P.G. Edwards 2008, The Parsec-Scale Jets of the TeV Blazars H 1426+428, IES 1959+650, and PKS 2155-304: 2001-2004, astro-ph/0801.2749.

[18] M. Punch (for the H.E.S.S. Collaboration) 2007, Long-Term VHE Gamma-Ray Monitoring of PKS 2155-304 with H.E.S.S. and Multiwavelength measurements, 2002-2005, in proceedings of International Cosmic Ray Conference 30 (799). 
[19] M. Sikora, M.C. Begelman, M.J. Rees 1994, Comptonization of diffuse ambient radiation by a relativistic jet: The source of gamma rays from blazars?, ApJ 421 (153).

[20] P.S. Smith 1996, The Optical and Ultraviolet Polarization of BL Lac Objects and OVV Quasars, in proceedings of Blazar Continuum Variability ASP Conf Series (110).

[21] C.M. Urry, A. Treves, L. Maraschi et al. 1997, Multiwavelength Monitoring of the BL Lacertae Object PKS 2155-304 in 1994 May. III. Probing the Inner Jet through Multiwavelength Correlations, ApJ 486 (799).

[22] M.J. Yuan, H. Tran, B. Wills and D. Wills 2001, The Physics of Blazar Optical Emission Regions. I. Alignment of Optical Polarization and the VLBI Jet, in proceedings of Blazar Demographics and Physics ASP Conf Series (227). 